\title{
Artérias e segmentos anátomo-cirúrgicos da glândula adrenal em fetos de búfalos (Bubalus bubalis)*
}

CORRESPONDENCETO: Maria Angélica Miglino Departamento de Cirurgia Faculdade de Medicina Veterinária e Zootecnia da USP Av. Prof. Dr. Orlando Marques de Paiva, 87 - Cidade Universitária Armando de Salles Oliveira - 05508-900 - São Paulo SP - Brasil

e-mail:miglino@usp.br

1 - Departamento de Cirurgia Faculdade de Medicina Veterinária e Zootecnia da USP - SP

2 - Universidade Estadual Paulista - UNESP, Araçatuba, SP 3-Departamento de Morfologia Escola Paulista de Medicina UNIFESP, São Paulo, SP Universidade de Santo Amaro. São Paulo, SP

\section{RESUMO}

As artérias e os segmentos anátomo-cirúrgicos das glândulas adrenais em 30 fetos de búfalos foram estudados mediante o preenchimento do sistema arterial de cada animal com látex-Neoprene corado e dissecação. Verificou-se que à direita, a glândula é irrigada por nove vasos diferentes, e à esquerda por oito fontes diversas. O número de segmentos anátomocirúrgicos variou de cinco a 11 , sendo que à direita o número mais freqüente foi de três $(60,0 \%)$ e à esquerda três ou quatro $(63,3 \%)$.

UNITERMOS: Artérias; Segmento; Glândulas adrenais; Búfalo

\section{INTRODUÇÃO}

D iante do interesse de reconhecer a eventual existência de segmentos anátomo-cirúrgicos e diante da lacuna que se nota na literatura sobre os vasos que nutrem as glândulas adrenais, resolvemos estudar sua anatomia em búfalos. Procuramos determinar a origem do suprimento sangüíneo das glândulas adrenais e a disposição das artérias no seu parênquima, com vistas a identificar sua segmentação vascular.

\section{MATERIAL E MÉTODO}

Foram estudados 30 fetos de búfalos, de 3 a 9 meses de vida intra- uterina. Os fetos (oito machos e 22 fêmeas) tiveram a aorta e seus ramos injetados com látex-Neoprene corado e foram fixados em solução aquosa de formol a 10\%. Em seguida, as adrenais foram dissecadas e seus vasos foram observados sob lupa.

Fizemos esquema da disposição dos vasos em cada glândula, com vistas à apresentação dos resultados e ilustração.

A exposição dos resultados segue a descrição da glândula feita por Godinho et al. ${ }^{2}$ (1981), segundo a qual a glândula adrenal direita é aproximadamente triangular, com base apoiada na veia cava caudal, enquanto que a adrenal esquerda, alongada e maior do que a direita, acha-se situada ventralmente à aorta, entre o rim esquerdo e o diafragma.

Com a finalidade de estabelecer os territórios vasculares e segmentos anátomo-cirúrgicos das glândulas adrenais consideramos para ambas (direita e esquerda) as seguintes regiões:

a. Região caudal-contígua ao pólo cranial do rim;

\footnotetext{
Trabalho financiado pela FAPESP (Fundação de Amparo a Pesquisa do Estado de São Paulo), projeto 92/4380-4.
}

b. Região medial-justaposta à aorta;

c. Região lateral-oposta à aorta;

d. Região cranial-oposta ao pólo renal.

\section{RESULTADOS}

I - Artérias da Glândula Adrenal Esquerda - Tab. 1 e 2 (Fig. 1)

A origem e $\mathrm{o}$ número das artérias da glândula adrenal esquerda foram os seguintes:

1. Da artéria renal esquerda (Figs. 1 e 3): 22 casos em 30, ou seja, $73,3 \% \pm 8,1$. Desses casos, correspondentes a 6 machos e 16 fêmeas, 2 fêmeas apresentaram 3 artérias, 4 animais ( 3 fềmeas e 1 macho) apresentaram 2 artérias e os animais restantes $(53,3 \%)$ apresentaram uma única artéria.

2. Da artéria mesentérica cranial (Fig. 1): 22 casos em 30, ou seja, $73,3 \% \pm 8,1$. Desses casos, correspondentes a 6 machos e 16 fềmeas, 3 fềmeas $(10,0 \%)$ apresentaram 3 artérias, 5 animais (3 fêmeas e 2 machos, $16,6 \%$ ) apresentaram 2 artérias e os 14 restantes $(46,6 \%)$ apresentaram uma única artéria.

3. Da artéria frênico-abdominal caudal: 21 casos em 30, ou seja, $70,0 \% \pm 8,4$. Desses casos, correspondentes a 4 machos e 17 fềmeas, 2 fềmeas $(6,6 \%)$ apresentaram 3 artérias, 9 animais (7 fềmeas e 2 machos, $30 \%$ ) apresentaram 2 artérias e os 10 animais restantes $(33,3 \%)$ apresentaram uma única artéria.

4. Da artéria celíaca (Figs. I e 3): 19 casos em 30, ou seja, $63,3 \%$ $\pm 8,8$. Desses casos, correspondentes a 4 machos e 15 fêmeas, uma fềmea $(3,3 \%)$ apresentou 4 artérias; 3 animais (2 fêmeas e um macho, $10 \%)$ apresentaram 3 artérias; 9 animais (8 fềmeas e 1 macho, 30,0\%) apresentaram 2 artérias e os 6 animais restantes $(20,0 \%)$ apresentaram uma única artéria. 
5. Da aorta (artéria adrenal): 13 casos em 30 , ou seja, $43,3 \% \pm 9,1$. Desses casos, correspondentes a 6 machos e 7 fêmeas, 2 fềmeas apresentaram 2 artérias e os animais restantes $(36,6 \%)$ apresentaram uma única artéria.

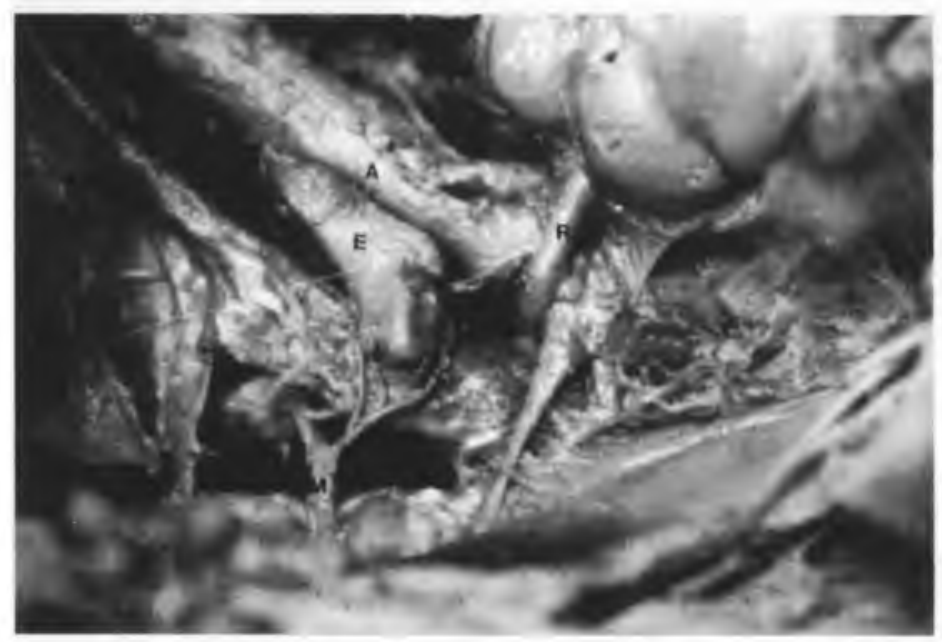

Figura 1

Fotografia de uma peça dissecada de um feto de búfalo, onde se vê a adrenal esquerda $(E)$, irrigada por ramos derivados das artérias celiaca $(C)$, aorta (artéria adrenal) $(A)$, mesentérica cranial $(M)$ e renal (R).

6. Da artéria costo-abdominal dorsal: 9 casos em 30, ou seja, $30,0 \% \pm 8,4$. Esses casos, correspondentes a 5 machos e 4 fêmeas, apresentaram uma única artéria.

7. Da primeira artéria lombar: 7 casos em 30 , ou seja, $23,3 \% \pm$ 7,7. Esses casos, correspondentes a 1 macho e 6 fêmeas, apresentaram uma única artéria.

8. Da décima segunda artéria intercostal: 3 casos em 30 , ou seja, $10,0 \% \pm 5,5$. Esses casos, correspondentes a 3 fềmeas, mostraram uma única artéria.

\section{II - Artérias da Glândula Adrenal Direita (Tab. 2) (Fig. 2)}

A origem e o número das artérias da adrenal direita foram os seguintes:

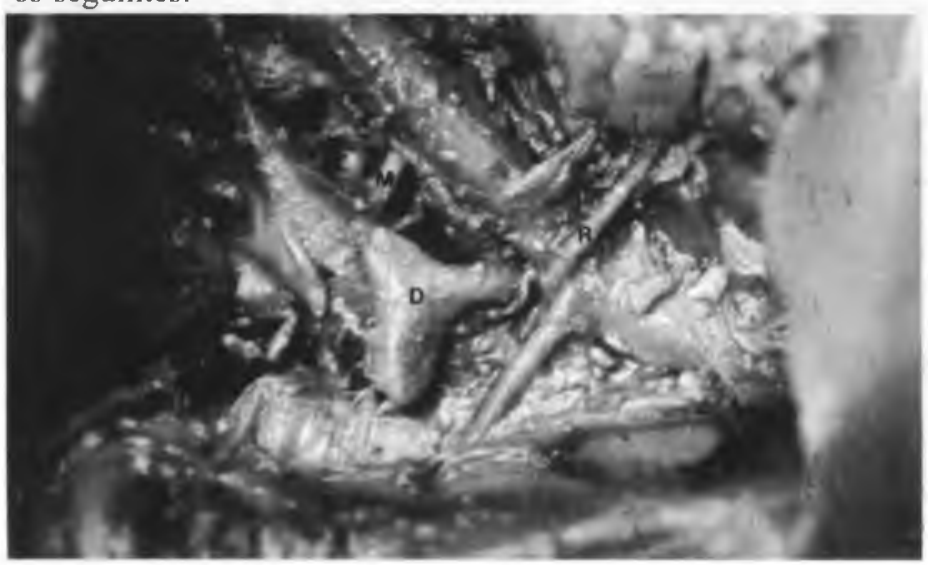

Figura 2

Fotografia de uma preparação de feto de búfalo, onde se nota a adrenal direita (D) recebendo vascularização da artéria renal (R) e das artérias celiaca $(C)$ e mesentérica $(M)$, quando estas surgem da aorta torácica. Nota-se o formato triangular ou estrelado da glândula direita.
1. Da artéria renal direita: 27 casos em 30 , ou seja, $90,0 \% \pm 5,5$. Desses casos, correspondentes a 8 machos e 19 fêmeas, 3 fêmeas $(10 \%)$ apresentaram 2 artérias e os animais restantes $(80 \%)$ apresentaram uma única artéria.

2. Da artéria frênico-abdominal caudal: 24 casos em 30, ou seja, $80,0 \% \pm 7,3$. Desses casos, correspondentes a 4 machos e 20 fêmeas, 4 animais $(13,3 \%)$ fêmeas apresentaram 3 artérias, 12 animais ( 9 fềmeas e 2 machos, $40,0 \%$ ) apresentaram duas artérias e os animais restantes $(26,6 \%)$ apresentaram uma única artéria.

3. Da aorta (artéria adrenal): 16 casos $\mathrm{em} 30$, ou seja, $53,3 \% \pm 9,1$. Desses casos, correspondentes a 4 machos e 12 fềmeas, I macho $(6,6 \%)$ apresentou 3 artérias; 1 macho $(6,6 \%)$ apresentou 2 artérias e os animais restantes $(46,6 \%)$ apresentaram uma única artéria.

4. Da artéria costo-abdominal dorsal: 7 casos em 30 , ou seja, $23,3 \%$ $\pm 7,7$. Desses casos, correspondentes a 4 machos e 3 fềmeas, 1 macho $(6,6 \%)$ apresentou 3 artérias; 1 fêmea $(6,6 \%)$ apresentou 2 artérias e os animais restantes $(46,6 \%)$ apresentaram uma única artéria.

Tabela 1

Origem das artérias penetrantes na glândula adrenal esquerda. São Paulo, 1997

\begin{tabular}{l|cccccccc}
\hline SEXO & \multicolumn{7}{|c}{ ORIGEM DO VASO E NUMERO DE VASOS } \\
\hline & AAA & ARE & ACC & AFC & AMC & AC & 12 IC & 1 AL \\
\hline $1 \mathrm{M}$ & 1 & - & - & - & - & 3 & - & - \\
$2 \mathrm{~F}$ & - & 1 & 1 & 1 & 3 & - & - & - \\
$3 \mathrm{~F}$ & 1 & - & 1 & 2 & 2 & 1 & - & - \\
$4 \mathrm{M}$ & 1 & 1 & 1 & - & 1 & 1 & - & - \\
$5 \mathrm{~F}$ & 1 & - & 1 & 1 & - & 2 & - & - \\
$6 \mathrm{M}$ & 1 & 1 & 1 & - & - & 2 & - & - \\
$7 \mathrm{~F}$ & 1 & - & - & 2 & 1 & - & 1 & - \\
$8 \mathrm{M}$ & 1 & 1 & 1 & - & 2 & 1 & - & - \\
$9 \mathrm{M}$ & - & 1 & 1 & 1 & 2 & - & - & - \\
$10 \mathrm{~F}$ & - & 1 & - & 1 & 1 & 2 & 1 & - \\
$11 \mathrm{M}$ & - & - & 1 & 1 & 1 & - & - & - \\
$12 \mathrm{M}$ & 1 & 1 & - & 2 & - & - & - & - \\
$13 \mathrm{~F}$ & - & - & 1 & - & 1 & 1 & - & - \\
$14 \mathrm{~F}$ & 2 & 1 & - & - & 1 & - & 1 & - \\
$15 \mathrm{~F}$ & 1 & 1 & - & 1 & 1 & - & - & - \\
$16 \mathrm{M}$ & 1 & 2 & - & 2 & 1 & - & - & 1 \\
$17 \mathrm{~F}$ & - & 1 & - & - & 3 & - & - & - \\
$18 \mathrm{~F}$ & 2 & 1 & - & - & 1 & 1 & - & 1 \\
$19 \mathrm{~F}$ & - & 3 & - & 2 & 2 & 1 & - & 1 \\
$20 \mathrm{~F}$ & - & 3 & - & 1 & - & 2 & - & - \\
$21 \mathrm{~F}$ & - & 1 & - & 3 & 3 & - & - & - \\
$22 \mathrm{~F}$ & 1 & - & - & 1 & - & 2 & - & 1 \\
$23 \mathrm{~F}$ & - & 1 & - & 1 & 2 & - & - & - \\
$24 \mathrm{~F}$ & - & 2 & - & 2 & 1 & 2 & - & 1 \\
$25 \mathrm{~F}$ & - & 2 & - & 2 & 1 & 3 & - & 1 \\
$26 \mathrm{~F}$ & - & 2 & - & 2 & - & 3 & - & - \\
$27 \mathrm{~F}$ & - & 1 & - & 2 & - & 2 & - & - \\
$28 \mathrm{~F}$ & - & 1 & - & - & 1 & 4 & - & - \\
$29 \mathrm{~F}$ & - & 1 & - & 3 & 1 & 2 & - & - \\
$30 \mathrm{~F}$ & - & - & - & 1 & 2 & 1 & - & 1 \\
\hline & & & & & & & & \\
\hline
\end{tabular}




\section{ABREVIAÇÕES USADAS NAS TABELAS}

$M$ - macho

F - fêmea

D - lado direito

E - lado esquerdo

Obs. - observação

seg. - segmento

AAA - Aorta (Artéria Adrenal)

ARE - Artéria Renal Esquerda

ARD - Artéria Renal Direita

ACC - Artéria Costo-Abdominal Caudal

AFC - Artéria Frênico-Abdominal Caudal

AMC - Artéria Mesentérica Cranial

AC - Artéria Celíaca

12 IC - Décima segunda Artéria Intercostal

11 IC - Décima primeira Artéria Intercostal

1-AL - Primeira Artéria Lombar

Tabela 2

Origem das artérias penetrantes na glândula adrenal direita. São Paulo, 1997

\begin{tabular}{|c|c|c|c|c|c|c|c|c|c|}
\hline \multirow[t]{2}{*}{ SEXO } & \multicolumn{9}{|c|}{ ORIGEM DO VASO E NÚMERO DE VASOS } \\
\hline & AAA & ARE & $\mathrm{ACC}$ & AFC & AMC & $\mathrm{AC}$ & $12 \mathrm{IC}$ & $1 \mathrm{IC}$ & $1 \mathrm{AL}$ \\
\hline $1 \mathrm{M}$ & - & 1 & - & - & 1 & 2 & - & - & - \\
\hline $2 F$ & - & 1 & 2 & - & - & 1 & - & 1 & - \\
\hline $3 F$ & 1 & 1 & - & 2 & - & - & - & - & - \\
\hline $4 M$ & 1 & 1 & 3 & - & - & - & - & - & - \\
\hline $5 F$ & - & 1 & 1 & 1 & - & - & - & - & - \\
\hline $6 \mathrm{M}$ & - & 1 & 1 & - & - & - & 1 & - & - \\
\hline $7 F$ & - & 1 & - & 1 & - & - & 1 & - & - \\
\hline $8 M$ & 1 & 11 & 1 & 1 & - & - & - & - & - \\
\hline $9 \mathrm{M}$ & 3 & 1 & 1 & - & - & - & - & - & - \\
\hline $10 \mathrm{~F}$ & - & 1 & 1 & - & - & 1 & 1 & - & - \\
\hline $11 \mathrm{M}$ & - & 1 & - & 2 & - & - & - & - & - \\
\hline $12 \mathrm{M}$ & 2 & 1 & - & 2 & - & - & - & - & - \\
\hline $13 F$ & - & 1 & - & 1 & - & 1 & 1 & - & - \\
\hline $14 \mathrm{~F}$ & - & 1 & - & 2 & - & - & - & - & - \\
\hline $15 F$ & - & 1 & - & 1 & - & - & - & - & - \\
\hline $16 \mathrm{M}$ & 1 & 1 & - & 1 & - & - & - & - & - \\
\hline $17 \mathrm{~F}$ & 1 & - & - & 3 & - & - & - & - & - \\
\hline $18 \mathrm{~F}$ & 1 & 1 & - & 2 & - & - & - & - & - \\
\hline $19 F$ & 1 & - & - & 2 & - & - & - & 1 & - \\
\hline $20 F$ & 1 & 2 & - & 2 & - & - & - & - & - \\
\hline $21 \mathrm{~F}$ & 1 & 2 & - & 2 & - & - & - & - & 1 \\
\hline $22 \mathrm{~F}$ & 1 & 1 & - & 2 & - & - & - & - & 1 \\
\hline $23 F$ & - & 1 & - & 2 & - & - & - & - & - \\
\hline $24 \mathrm{~F}$ & - & 1 & - & 2 & 1 & 1 & - & - & 1 \\
\hline $25 \mathrm{~F}$ & 2 & 2 & - & 2 & - & - & - & - & - \\
\hline $26 \mathrm{~F}$ & 2 & - & - & 1 & - & - & - & - & 2 \\
\hline $27 \mathrm{~F}$ & 1 & 1 & - & 3 & - & - & - & - & - \\
\hline $28 \mathrm{~F}$ & - & 1 & - & 3 & - & - & - & - & - \\
\hline $29 \mathrm{~F}$ & 1 & 1 & - & 3 & - & - & - & - & - \\
\hline $30 F$ & 2 & 1 & - & 1 & - & - & - & - & - \\
\hline
\end{tabular}

5. Da artéria celialea: 5 casos em 30, ou seja, 16,6\% 10,8 . Desses casos, correspondentes a 1 macho e 4 fêmeas, o macho $(3,3 \%)$ apresentou 2 artérias. Os animais restantes apresentaram uma unica artéria.

6. Da décima segunda artéria intercostal: 4 casos em 30, ou seja. $13,3 \% \pm 6,2$. Lisses casos, correspondentes a 1 macho e 3 femcals. apresentaram uma única artéria.

7. Da primcira artéria lombar: 4 casos em 30, ou seja. 13.3\% 10.2 . Desses casos, correspondentes a 4 femeas. I fêneal apresentou $\left(3,3^{\circ} \%\right)$ apresentou 2 artérias e os animais restantes apresentaram uma únical artéria.

8. Da artéria mesentérica cranial: 2 casos em 30, ou seja, 6,6\% 4.5. Esses casos, correspondentes a 2 remeas, apresentaram umal unica alrtéria.

9. Da décima primeira artéria intercostal: 2 casos em 30, ou sejal, $6.6 \%+4.5$. Esses calsos, correspondentes a 2 fêmeas, apresentaram uma única artéria.

\section{SEGMENTOS ANÁTOMO-CIRÚRCIICOS DAS (ILANNI)ULAS ADRENAIS}

Foram encontrados segmentos anátomo-cirúrgicos bilateralmente em todos os casos. O número de segmentos foi igual biliteralmente em 6 casos $(20.0 \%+7.3)$ e diferente em 24 calsos $\left(80,()_{0}^{\circ} \pm 7,3\right)$. (Fig. 3).

1. O numero máximo de segmentos em cadal caso (somados os da glândula adrenal direila ea esquerda) for de 14 (em 2 casos) e o minimo foi de 6 ( 3 calsos), de acordo com a seguinte distribuição (Tab.3):

Notil-se yuc o numero total mais alto de segmentos é 8 ou 10, que ocorreu em 7 dos casos.

Tanto a igualdade quanto a diferença bilateral do numero de segmentos foi notada em fêmeas e machos.

O número de segmentos foi igual bilateralmente em 8 cassos; foi maior à esquerda em 18 c major à direita em 4 cassos.

2. () número de segmentos à direila variou de 2 a 7 e sua distribuição foi a seguinte (Tab.4):

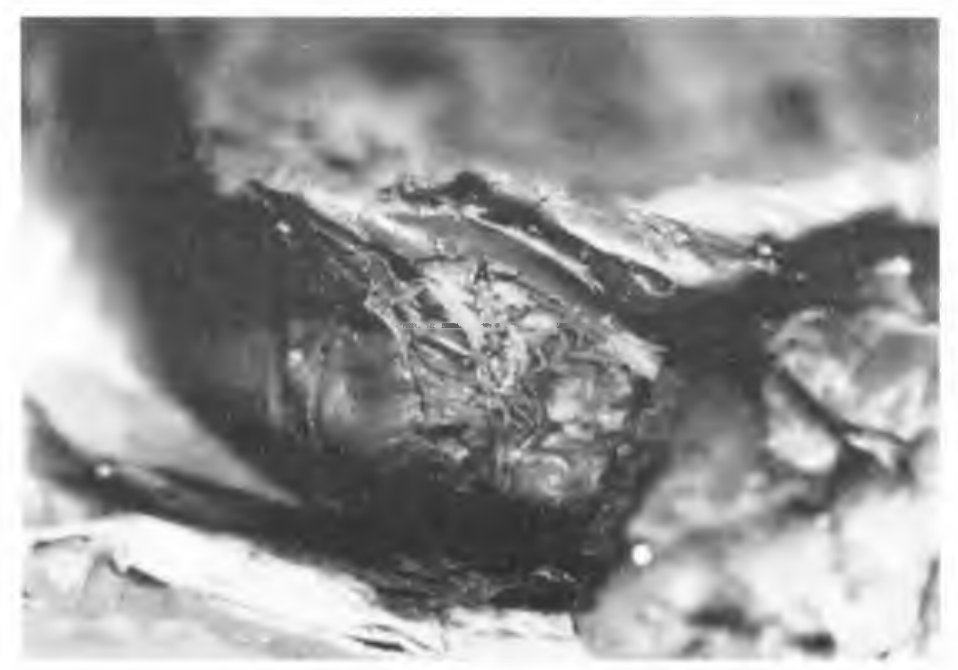

Figura 3

Fotografia da dissecação interna da adrenal direita de búfalo, mostrando a predominância da artéria adrenal direita (A) vascularizando a glândula. 


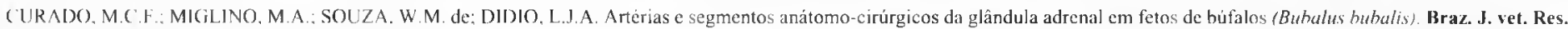
anim. Sci. São Paulo, v.34. n.3. p. 125-130, 1997

Tabela 3

Número de segmentos anátomo-cirúrgicos nas adrenais direita e esquerda. São Paulo, 1997

\begin{tabular}{c|c}
\hline $\begin{array}{c}\text { NÚMERO } \\
\text { DE CASOS }\end{array}$ & $\begin{array}{c}\text { NÚMERO DE SEGMENTOS } \\
\text { ANÁTOMO-CIRÚRGICOS }\end{array}$ \\
\hline 6 & $3(10,0 \% \pm 5,5)$ \\
7 & $1(3,3 \% \pm 3,3)$ \\
8 & $7(23,3 \% \pm 7,7)$ \\
9 & $4(13,3 \% \pm 6,2)$ \\
10 & $7(23,3 \% \pm 7,7)$ \\
11 & $5(16,6 \% \pm 6,8)$ \\
13 & $1(3,3 \% \pm 3,3)$ \\
14 & $2(6,6 \% \pm 4,5)$ \\
\hline
\end{tabular}

O número mais alto de segmentos da glândula adrenal direita foi 4 , que ocorreu em 12 casos $(40,0 \% \pm 8,9)$.

3. O número de segmentos à esquerda variou de 3 ( 2 casos) a 9 ( 1 caso) e sua distribuição foi a seguinte (Tab.5):

O número mais alto de segmentos anátomo-cirúrgicos da glândula adrenal esquerda foi de 4 , que ocorreu $\mathrm{cm} 9$ casos $(30,0 \%$ $+8,4)$.

4. A distribuição dos casos pelo número de segmentos em cada lado é seguinte (Tab.6):

$\Lambda$ disposição mais freqüente é a de quatro segmentos à direita e scis segmentos à esquerda ( 5 casos, ou seja, 16,6\% $\pm 6,8$ ) c a de 4 segmentos em cada lado (4 casos, ou seja, 13,3\% $\pm 6,2$ ).

Tabela 4

Número de segmentos anátomo-cirúrgicos à direita. São Paulo, 1997

\begin{tabular}{c|c}
\hline $\begin{array}{c}\text { NÚMERO } \\
\text { DE CASOS }\end{array}$ & $\begin{array}{c}\text { NÚMERO DE SEGMENTOS } \\
\text { ANÁTOMO-CIRÚRGICOS }\end{array}$ \\
\hline 2 & $1(3,3 \% \pm 3,3)$ \\
3 & $7(23,3 \% \pm 7,7)$ \\
4 & $12(40,0 \% \pm 8,9)$ \\
5 & $6(20,0 \% \pm 7,3)$ \\
7 & $1(3,3 \% \pm 3,3)$ \\
\hline
\end{tabular}

\section{COMENTÁRIOS}

As artérias da adrenal de ruminantes foram estudadas por vários autores tais como Harrison; Mc Donald ${ }^{3}$ (1966); Russo; Pelagalli' (1972): Godinho ${ }^{2}$ (1981); Getty' (1981); Tewari; Prakashi ${ }^{6}$

Tabela 5

Número de segmentos anátomo-cirúrgicos à esquerda. São Paulo, 1997

\begin{tabular}{c|c}
\hline $\begin{array}{c}\text { NÚMERO } \\
\text { DE CASOS }\end{array}$ & $\begin{array}{c}\text { NÚMERO DE SEGMENTOS } \\
\text { ANÁTOMO-CIRÚRGICOS }\end{array}$ \\
\hline 3 & $2(6,6 \% \pm 4,5)$ \\
4 & $9(30,0 \% \pm 8,4)$ \\
5 & $7(23,3 \% \pm 7,7)$ \\
6 & $7(23,3 \% \pm 7,7)$ \\
7 & $3(10,0 \% \pm 5,5)$ \\
8 & $1(3,3 \% \pm 3,3)$ \\
9 & $1(3,3 \% \pm 3,3)$ \\
\hline
\end{tabular}

Tabela 6

Distribuição dos casos pelo número de segmentos. São Paulo, 1997

\begin{tabular}{c|c}
\hline $\begin{array}{c}\text { NÚMERO } \\
\text { DE CASOS }\end{array}$ & $\begin{array}{c}\text { NÚMERO DE SEGMENTOS } \\
\text { ANÁTOMO-CIRÚRGICOS }\end{array}$ \\
\hline 2D-4E & $1(3,3 \% \pm 3,3)$ \\
3D-3E & $2(6,6 \% \pm 4,5)$ \\
3D-4E & $1(3,3 \% \pm 3,3)$ \\
$3 D-5 E$ & $3(10,0 \% \pm 5,5)$ \\
3D-6E & $1(3,3 \% \pm 3,3)$ \\
4D-4E & $1(13,3 \% \pm 6,2)$ \\
4D-5E & $1(3,3 \% \pm 3,3)$ \\
$4 D-6 E$ & $5(16,6 \% \pm 6,8)$ \\
$4 D-7 E$ & $2(6,6 \% \pm 4,5)$ \\
5D-4E & $2(6,6 \% \pm 4,5)$ \\
5D-5E & $2(6,6 \% \pm 4,5)$ \\
5D-6E & $1(3,3 \% \pm 3,3)$ \\
5D-9E & $1(3,3 \% \pm 3,3)$ \\
6D-5E & $1(3,3 \% \pm 3,3)$ \\
6D-7E & $1(3,3 \% \pm 3,3)$ \\
6D-8E & $1(3,3 \% \pm 3,3)$ \\
$6 D-4 E$ & $1(3,3 \% \pm 3,3)$ \\
\hline
\end{tabular}

(1988) e por Miglino et al. ${ }^{4}$ (1995). Entretanto, os dois primeiros trabalhos foram dedicados aos pequenos ruminantes fovinos $\mathrm{e}$ caprinos), razão pela qual comentaremos nossos resultados somente em função dos resultados encontrados por Godinho et $a l^{2}$ (1981); Getty (1981); Tewari; Prakashib (1988) e por Miglino et al. ${ }^{4}$ (1995), que pesquisaram este assunto nos grandes ruminantes (bovinos e bubalinos).

De acordo com Godinhoet al. ${ }^{2}(1981)$, as glândulas adrenais são irrigadas nos bovinos por ramos das artérias frênicas caudais, celíaca e renais, sem entretanto especificar as percentagens de participação desses vasos à direita e à esquerda.

Para Getty' (1981), nos bovinos cada adrenal recebe duas artérias, ou da aorta ou de ramos das artérias renais, porém estes dados, quando confrontados com os apresentados por Tewari; Prakashí ${ }^{6}$ (1988) nos búfalos, são igualmente incompletos, já que para eles o ramo adrenal cranial por vezes vem da artéria celíaca.

É certo que Miglino et al. ${ }^{4}$ (1995) encontraram em bovinos azebuados 8 fontes diferentes das artérias dirigidas à adrenal direita e 10 à csquerda e que a participação das artérias frênicas caudais e renais foram constantes à direita $(66,3 \%$ c $50,0 \%)$ e à esquerda $(3,3 \%$ e $43,3 \%)$, respectivamente

Quanto à participação de ramos derivados da artéria celíaca, este arranjo foi observado nos bovinos azebuados somente à esquerda $(36,6 \%)$.

Encontramos nos búfalos nove fontes diferentes de origem de vasos endereçados à adrenal direita $\mathrm{e}$ a oito à esquerda (Tabs 9 e 10).

As Tabs. 7 e 8 mostram uma comparação das fontes de origem dos vasos da adrenal nos bovinos azebulados (Miglino et al. $\left.{ }^{4}, 1995\right)$ e nos búfalos.

E notável o número de vasos das glândulas adrenais nos bovinos e a participação de diversas fontes de origem desses ramos. E nossa intenção analisar $\mathrm{cm}$ pesquisas futuras o comportamento da drenagem venosa das adrenais e verificar a eventual multiplicidade 
Tabela 7

Número total e denominação dos segmentos da adrenal direita em búfalos (Bubalus bubalis). São Paulo, 1997

\begin{tabular}{|c|c|}
\hline SEXO & $\begin{array}{c}\text { NÚMERO TOTAL DE DENOMINAÇÃO } \\
\text { DOS SEGMENTOS DA } \\
\text { GLÂNDULA ADRENAL DIREITA }\end{array}$ \\
\hline $1 \mathrm{M}$ & 5 segm ( 1 caudal, 2 craniais, 1 lateral, 1 medial) \\
\hline $2 \mathrm{~F}$ & 5 segm ( 1 caudal, 2 craniais, 1 lateral, 1 mediai) \\
\hline $3 \mathrm{~F}$ & 4 segm ( 1 caudal, 1 cranial, 1 lateral, 1 medial) \\
\hline $4 \mathrm{M}$ & 6 segm ( 1 caudal, 2 craniais, 1 lateral, 1 medial) \\
\hline $5 \mathrm{~F}$ & 3 segm ( 1 caudal, 1 crânio-medial, 1 lateral) \\
\hline $6 \mathrm{M}$ & 3 segm ( 1 crânio-médio-lateral, 1 lateral) \\
\hline $7 F$ & 3 segm ( 1 caudal, 1 cranial, 1 lateral) \\
\hline $8 \mathrm{M}$ & 3 segm ( 1 caudal, 1 crânio-medial, 1 lateral) \\
\hline $9 \mathrm{M}$ & 5 segm ( 1 caudal, 1 cranial, 1 lateral, 2 mediais) \\
\hline $10 \mathrm{~F}$ & 4 segm ( 1 caudal, 2 craniais, 1 lateral) \\
\hline $11 \mathrm{M}$ & 3 segm ( 1 caudal, 1 crânio-medial, 1 lateral-cranial) \\
\hline $12 \mathrm{M}$ & 5 segm ( 1 caudal, 1 cranial, 1 lateral, 2 mediais) \\
\hline $13 \mathrm{~F}$ & 3 segm ( 1 caudal, 1 crânio-medial, 1 lateral) \\
\hline $14 \mathrm{~F}$ & 3 segm ( 1 caudal, 2 craniais) \\
\hline $15 \mathrm{~F}$ & 2 segm ( 1 caudal, 1 lateral) \\
\hline $16 \mathrm{M}$ & 4 segm ( 1 caudal, 2 mediais, 2 médio-crânio-lateral) \\
\hline $17 \mathrm{~F}$ & 4 segm ( 1 cranial, 1 crânio-medial, 1 lateral, 1 medial) \\
\hline $18 \mathrm{~F}$ & $\begin{array}{l}4 \text { segm ( } 1 \text { lateral, } 1 \text { cranial, } 1 \text { lateral, } 1 \text { medial, } \\
1 \text { médio-caudo-lateral) }\end{array}$ \\
\hline $19 \mathrm{~F}$ & 4 segm ( 1 cranial, 1 medial, 2 crânio-mediais) \\
\hline $20 \mathrm{~F}$ & 4 segm ( 2 craniais, 2 mediais) \\
\hline $21 \mathrm{~F}$ & 4 segm (2 caudais, 2 laterais, 1 medial) \\
\hline $22 \mathrm{~F}$ & 4 segm ( 1 caudal, 1 cranial, 1 medial, 1 lateral) \\
\hline $23 \mathrm{~F}$ & 4 segm ( 1 caudal, 1 lateral, 2 mediais) \\
\hline $24 \mathrm{~F}$ & 6 segm (1 caudal, 3 craniais, 1 lateral, 1 medial) \\
\hline $25 \mathrm{~F}$ & 5 segm ( 1 caudal, 2 craniais, 2 mediais) \\
\hline $26 \mathrm{~F}$ & 7 segm ( 1 caudal, 3 craniais, 1 lateral, 2 mediais) \\
\hline $27 \mathrm{~F}$ & 5 segm ( 1 caudal, 1 cranial, 1 lateral, 2 mediais) \\
\hline $28 \mathrm{~F}$ & 4 segm ( 1 caudal, 1 cranial, 1 lateral, 1 medial) \\
\hline $29 \mathrm{~F}$ & $\begin{array}{l}6 \text { segm ( } 1 \text { caudal, } 1 \text { cranial, } 1 \text { lateral, } 2 \text { mediais, } \\
1 \text { crânio-medial) }\end{array}$ \\
\hline $30 \mathrm{~F}$ & 4 segm ( 1 caudal, 1 cranial, 2 mediais) \\
\hline
\end{tabular}

e diversidade de origem.

Analisando agora a segmentação arterial das glândulas, verificamos que foram encontrados segmentos anátomo-cirúrgicos bilateralmente, tanto nos bovinos quanto nos bubalinos. O número de segmentos foi igual bilateralmente em $23,3 \%$ e $20,0 \%$ e diferente em 76,6\% e $80,0 \%$ nos bovinos e bubalinos, respectivamente.

O número máximo de segmentos (soma das adrenais direita e esquerda) foi $11(36,6 \%)$ nos bovinos azebuados e $14(46,6 \%)$ e o mínimo foi $5(16,6 \%)$ nos bovinos azebuados e $6(20,0 \%)$ nos bubalinos.

Nos bovinos Azebuados o número de segmentos foi maior à esquerda em $53,3 \%$ dos casos, contra $60,0 \%$ dos casos dos bubalinos; foi maior à direita $\mathrm{em} 47,7 \%$ dos casos nos bovinos e em $13,3 \%$ dos casos nos bubalinos.

A direita, o número de segmentos variou de dois $(6,6 \%)$ a seis $(3,3 \%)$, mais freqüentemente $3(60,0 \%)$ nos bovinos, e de 2 $(3,3 \%)$ a $7(3,3 \%)$, mais freqüentemente $4(40,0 \%)$ nos bubalinos.

Á esquerda, o número de segmentos variou de $2(13,3 \%)$ a
Tabela 8

Número total e denominação dos segmentos da adrenal esquerda em búfalos (Bubalus Bubalis). São Paulo, 1997

\begin{tabular}{|c|c|}
\hline SEXO & $\begin{array}{c}\text { NÚMERO TOTAL DE DENOMINAÇÃO } \\
\text { DOS SEGMENTOS DA } \\
\text { GLÂNDULA ADRENAL ESQUERDA }\end{array}$ \\
\hline $1 \mathrm{M}$ & 4 segm ( 1 caudal, 1 cranial, 1 lateral, 1 crânio-medial) \\
\hline $2 F$ & 6 segm ( 1 caudal, 2 craniais, 2 laterais, 1 medial) \\
\hline $3 F$ & 6 segm ( 1 caudal, 2 craniais, 2 laterais, 1 medial) \\
\hline $4 \mathrm{M}$ & 5 segm ( 1 caudal, 1 cranial, 2 laterais, 1 medial) \\
\hline $5 \mathrm{~F}$ & 5 segm ( 1 caudal, 1 crânio-medial, 2 laterais) \\
\hline $6 \mathrm{M}$ & $\begin{array}{l}5 \text { segm ( } 1 \text { caudal, } 1 \text { médio-caudo-lateral, } 2 \text { craniais, } \\
1 \text { lateral) }\end{array}$ \\
\hline $7 F$ & 4 segm ( 1 caudal, 2 craniais, 1 lateral) \\
\hline $8 \mathrm{M}$ & $\begin{array}{l}6 \text { segm ( } 1 \text { caudal, } 2 \text { craniais, } 2 \text { laterais, } \\
1 \text { caudo-medial) }\end{array}$ \\
\hline $9 \mathrm{M}$ & 5 segm ( 1 caudal, 2 craniais, 1 lateral, 2 crânio-medial) \\
\hline $10 \mathrm{~F}$ & 6 segm ( 1 caudal, 2 craniais, 3 laterais) \\
\hline $11 \mathrm{M}$ & 3 segm (1 médio-caudal, 1 cranial, 1 lateral) \\
\hline $12 \mathrm{M}$ & 4 segm ( 2 caudais, 1 crânio medial, 1 crânio-lateral) \\
\hline $13 \mathrm{~F}$ & 3 segm (1 cranial, 1 medial, 1 lateral) \\
\hline $14 \mathrm{~F}$ & 5 segm ( 3 caudais, 3 craniais) \\
\hline $15 \mathrm{~F}$ & 4 segm ( 2 caudais, 2 craniais) \\
\hline $16 \mathrm{M}$ & $\begin{array}{l}6 \text { segm ( } 1 \text { caudal, } 3 \text { craniais, } 1 \text { lateral, } \\
1 \text { caudo-medial) }\end{array}$ \\
\hline $17 \mathrm{~F}$ & 4 segm ( 1 caudal, 2 craniais, 1 lateral) \\
\hline $18 \mathrm{~F}$ & 6 segm ( 3 caudais, 1 cranial, 2 laterais) \\
\hline $19 \mathrm{~F}$ & 7 segm ( 1 cranial, 3 craniais, 2 laterais, 1 medial) \\
\hline $20 \mathrm{~F}$ & 4 segm ( 1 caudal, 2 craniais, 1 lateral) \\
\hline $21 \mathrm{~F}$ & 6 segm ( 1 caudal, 2 craniais, 3 laterais) \\
\hline $22 \mathrm{~F}$ & 5 segm ( 1 caudal, 2 craniais, 2 laterais) \\
\hline $23 F$ & 4 segm ( 1 caudal, 3 craniais) \\
\hline $24 \mathrm{~F}$ & 8 segm ( 2 caudais, 2 craniais, 3 laterais, 1 medial) \\
\hline $25 \mathrm{~F}$ & 9 segm (2 caudais, 2 craniais, 3 laterais, 2 mediais) \\
\hline $26 \mathrm{~F}$ & 4 segm ( 2 caudais, 2 craniais) \\
\hline $27 \mathrm{~F}$ & 5 segm ( 1 caudal, 2 craniais, 1 lateral, 1 medial) \\
\hline $28 \mathrm{~F}$ & 4 segm ( 1 caudal, 3 craniais) \\
\hline $29 \mathrm{~F}$ & 7 segm ( 1 caudal, 3 craniais, 2 laterais, 1 medial) \\
\hline $30 \mathrm{~F}$ & 7 segm ( 2 caudais, 3 craniais, 1 lateral, 1 medial) \\
\hline
\end{tabular}

Tabela 9

Origem dos ramos da artéria adrenal direita. São Paulo, 1997

\begin{tabular}{l|c|c}
\hline \multicolumn{1}{c|}{ ORIGEM } & $\begin{array}{c}\text { BOVINOS } \\
\text { AZEBUADOS }\end{array}$ & BÚFALOS \\
\hline Artéria Renal Direita & $50,0 \%$ & $90,0 \%$ \\
Artéria Frênico-Abdominal Caudal & $63,3 \%$ & $80,0 \%$ \\
Aorta (Artéria Adrenal) & $60,0 \%$ & $53,3 \%$ \\
Artéria Costo-Abdominal Caudal & $56,6 \%$ & $23,3 \%$ \\
Artéria Celiaca & - & $16,6 \%$ \\
I - Artéria Lombar & $56,6 \%$ & $13,3 \%$ \\
Artéria Mesentérica Cranial & - & $6,6 \%$ \\
II - Artéria Renal Acessória & $3,3 \%$ & - \\
Ramo Frênico Torácico & $3,3 \%$ & - \\
2. Artéria Lombar & $16,6 \%$ & - \\
\hline
\end{tabular}


Tabela 10

Origem dos ramos da artéria adrenal esquerda. São Paulo, 1997

\begin{tabular}{l|c|c}
\hline \multicolumn{1}{c|}{ ORIGEM } & $\begin{array}{c}\text { BOVINOS } \\
\text { AZEBUADOS }\end{array}$ & BÚFALOS \\
\hline Artéria Renal Esquerda & $43,3 \%$ & $73,3 \%$ \\
Artéria Mesentérica Cranial & $43,3 \%$ & $73,3 \%$ \\
Artéria Frênico-Abdominal Caudal & $63,3 \%$ & $70,0 \%$ \\
Artéria Celiaca & $36,6 \%$ & $63,3 \%$ \\
Aorta (Artéria Adrenal) & $66,6 \%$ & $43,3 \%$ \\
1 - Artéria Lombar & $83,3 \%$ & $23,3 \%$ \\
12 - Artéria Intercostal & - & $10,0 \%$ \\
Tronco Celiaco Mesentérico & $3,3 \%$ & - \\
2 - Artéria Lombar & $26,6 \%$ & - \\
3 - Artéria Lombar & $3,3 \%$ & - \\
\hline
\end{tabular}

$8(3,3 \%)$, mais freqüentemente 3 ou $4(33,3 \%$ e $30,0 \%)$ nos bovinos, e de $3(6,6 \%)$ a $9(3,3 \%)$, mais freqüentemente 5 ou $6(30,0 \%)$ nos bubalinos.

A disposição mais freqüente para os bovinos é a de 3 segmentos à direita e 4 à esquerda $(23,3 \%)$ e de 3 segmentos de cada lado $(20,0 \%)$. Nos bubalinos a disposição mais freqüente é de 4 segmentos à direita e 6 segmentos à esquerda $(16,6 \%)$ e a de 4 segmentos de cada lado $(13,3 \%)$.

\section{CONCLUSÕES}

1. A adrenal direita do búfalo recebe vasos derivados de 9 fontes diferentes à direita e 8 à esquerda.

2. Foram encontrados segmentos anátomo-cirúrgicos nas adrenais dos bubalinos. O número de segmentos foi igual bilateralmente em $20,0 \%$ e diferente em $80,0 \%$ dos casos.

3. O número máximo de segmentos foi igual a $14(46,6 \%)$ e o mínimo $6(20,0 \%)$.

4. O número de segmentos anátomo-cirúrgicos foi maior à esquerda $(60,0 \%)$ e menor à direita $(13,3 \%)$ nos bubalinos.

5. Á direita o número de segmentos variou de $2(3,3 \%)$ a $7(3,3 \%)$, mais freqüentemente 5 ou 6 .

6. Ȧ esquerda o número de segmentos variou de $3(6,6 \%)$ a 9 $(6,6 \%)$, mais freqüentemente 5 ou $6(30,0 \%)$.

7. Nos bubalinos a disposição mais freqüente é de 4 segmentos à direita e 6 segmentos à esquerda.

\section{SUMMARY}

The arteries and the anatomico-surgical segments of the adrenal glands of 30 fetuses of buffaloes were studied after arterial injection with colored latex-Neoprene and dissection. The right gland, in our cases, appeared to be supplied by 9 , and the left by 8 branches, from different sources and showing different arrangements. The anatomicosurgical segments varied in number, from 5 to 11 , the average being three on the right $(60,0 \%)$, and 3 or 4 on the left $(63,3 \%)$.

UNITERMS: Arteries; Segments; Adrenal glands; buffaloes

\section{REFERÊNCIAS BIBLIOGRÁFICAS}

I- GETTY, R. Anatomia dos animais domésticos. 5. ed. Rio de Janeiro. Interamericana, 1981.v. 1. p. 898 .

2- GODINHO, H.P.; CARDOSO, F.M.; NASCIMENTO, J.F. Anatomia dos ruminantes domésticos. Belo Horizonte, Instituto de Ciências Biológicas, 1981. p.361

3-HARRISON, F.A.: MCDONALD, I.R.: The arterial suply to the adrenal gland of the sheep. Journal of Anatomy, v. 100, n. I, p. 189-202, 1966.

4- MIGLINO, M.A.; DIDIO, L.J.A.; COSTA, J.B.; PACHECO, Y.G. Amérias c segmentos anátomo-cirúrgicos da adrenal $\mathrm{em}$ fetos de bovinos. Brazilian Journal of Veterinary Reserch and Animal Science, v.32, n.2, p.75-82, 1995.
5-RUSSO, E.; PELAGALLI, G.V. Macro-et micro-circulation des glandes surrénales chez les petits ruminants. Recherches effectuées sur les espèces ovines et caprines. Acta Anatomica, v.82, n.2, p.179-97, 1972.

6- TEWARI, A.N.; PRAKASHI, P. A note on the blood supply to the adrenal gland and the spleen in the buffalo. Indian Veterinary Journal, v. 65, n. 3, p. 270 . 1988
Recebido para publicação: 18/1/96 Aprovado para publicação: 3/7/97 